\title{
Ulcerative Colitis in the Elderly
}

\section{Silvestro Canonico*, Gianluca Pellino and Francesco Selvaggi}

Department of Medical, Surgical, Neurological, Metabolic and Ageing Sciences, Second University of Naples, Italy

Ulcerative colitis (UC) is an inflammatory bowel disease involving the mucosal surface of the large bowel, usually starting from the rectum and extending proximally in a continuous fashion through the entire colon $[1,2]$. Restorative proctocolectomy with ileal pouch and ileal pouch-anal anastomosis (IPAA) is the treatment of choice for refractory or complicated UC, removing the entire diseased mucosa [2-5]. IPAA controls some extra-intestinal manifestations of UC and almost eliminates the risk for UC-related colorectal cancer [6].

Whether IPAA should be offered to elderly patients still remains debated. Guidelines on IPAA in elderly patients are definitively lacking in the literature. Older patients are often found with comorbidities, which may increase the risk of postoperative complications. Also, the physiological decrease in sphincter function may lead to worse bowel control.

First of all, literature seems to lack a definition of "elderly" patients. The concept is not clearly stated in papers dealing with IPAA for UC. The most cited papers on the topic are those provided by Farouk et al. [7] and Delaney et al. [8]. The former study found patients undergoing IPAA in the elderly suffering from worse function [7], and these data were confirmed by the latter [8], although differences seemed less apparent at 10-year follow-up. However, they consider patients as "in the elderly" if over 45 or 50 years of age. It is our opinion that a cutoff value of at least 70 years of age - adjusting it on the basis of life expectancy - is more likely including patients who are actually in an elderly condition (i.e. not able to cope with work activity), reflecting an higher rate of comorbidities and need for concomitant medications.

We have recently reported on our experience with IPAA in patients aged over 70 years [9]. Although such individuals are more often found with co-morbidities and frail, an accurate patient selection and a keen surgical technique can provide optimal functional results and high quality of life [9].

However, some facets need to be elucidated in elderly patients suffering from UC considered for IPAA. First, patients should not show clinical continence disturbances. Instrumental diagnostics do not seem useful routinely. Patients should be able to cope with everyday activity and active. Also, they should be highly motived and able to understand the procedure, adapting to their new anatomy and function.

Surgical variables which need to be taken into account consist of extensive experience with pelvic pouch procedure, performing IPAA in 2 or 3 stages (which mean that a diverting stoma should always be temporarily provided). Intra-operative complications should be carefully avoided as they may impact on future function [4]. During the peri-operative period, these should be promptly suspected and treated. Reintroduction on hypotensive agents should be done carefully in patients with arterial hypertension, as IPAA may lower pressure values; moreover the stoma output should be carefully recorded as the thirst mechanism is less effective in the elderly, potentially leading to dehydration- even requiring re-hospitalization. The stoma should be reversed at the soonest.

Conversely- when the above described conditions are met-elderly patients receiving IPAA benefit from an excellent bowel control and this reflects on high quality of life and social function [9]. Moreover, they are able to withdraw UC drug intake. Attention should be laid on post-operative surveillance: this should be carried out by means of flexible endoscopy performed yearly starting from ten years after the diagnosis of UC (not from IPAA) [6]

In conclusion, in selected patients, and in experienced hands, IPAA should be recommended also over 70 years of age; when ideal conditions are met, there are no reasons to expect worse results than those observed in younger patients.

\section{References}

1. Silverberg MS, Satsangi J, Ahmad T, Arnott ID, Bernstein CN, et al. (2005) Toward an integrated clinical, molecular and serological classification of inflammatory bowel disease: Report of a Working Party of the 2005 Montreal World Congress of Gastroenterology. Can J Gastroenterol 19: 5A-36A.

2. Selvaggi F, Giuliani A, Sciaudone G, Riegler G, Di Stazio C (2004) Quality of life in patients with ileal pouch for ulcerative colitis. Chir Ital 56: 239-246.

3. Pellino G, Sciaudone G, Canonico S, Selvaggi F (2012) Role of ileostomy in restorative proctocolectomy. World J Gastroenterol 18: 1703-1707.

4. Selvaggi F, Sciaudone G, Limongelli P, Di Stazio C, Guadagni I, et al. (2010) The effect of pelvic septic complications on function and quality of life after ileal pouch-anal anastomosis: a single center experience. Am Surg 76: 428-435.

5. Selvaggi F, Cuocolo A, Giuliani A, Sciaudone G, Riegler G, et al. (2006) The role of scintigraphic defecography in the assessment of bowel function afte restorative proctocolectomy for ulcerative colitis. Int J Colorectal Dis 21: 448452.

6. Egan L, D'Inca R, Jess T, Pellino G, Carbonnel F, et al. (2013) Non-colorecta intestinal tract carcinomas in inflammatory bowel disease: Results of the 3rd ECCO Pathogenesis Scientific Workshop (II). J Crohns Colitis.

7. Farouk R, Pemberton JH, Wolff BG, Dozois RR, Browning S, et al. (2000) Functional outcomes after ileal pouch-anal anastomosis for chronic ulcerative colitis. Ann Surg 231: 919-926.

8. Delaney CP, Fazio VW, Remzi FH, Hammel J, Church JM, et al. (2003) Prospective, age-related analysis of surgical results, functional outcome, and quality of life after ileal pouch-anal anastomosis. Ann Surg 238: 221-228.

9. Pellino G, Sciaudone G, Candilio G, Camerlingo A, Marcellinaro R, et al. (2013) Complications and functional outcomes of restorative proctocolectomy for ulcerative colitis in the elderly BMC Surg 13: S9.

*Corresponding author: Silvestro Canonico, Department of Medical, Surgical, Neurological, Metabolic and Ageing Sciences, Second University of Naples, Piazza Miraglia 5, 80138 Naples, Italy, Tel: 39-81-5665278; Fax: 39-81-5665278; E-mail: silvestro.canonico@unina2.it

Received November 14, 2013; Accepted November 16, 2013; Published November 16, 2013

Citation: Canonico S, Pellino G, Selvaggi F (2013) Ulcerative Colitis in the Elderly. J Gerontol Geriat Res 3: e125. doi:10.4172/2167-7182.1000e125

Copyright: (c) 2013 Canonico S, et al. This is an open-access article distributed under the terms of the Creative Commons Attribution License, which permits unrestricted use, distribution, and reproduction in any medium, provided the original author and source are credited. 